\title{
Wang-Tiles for the Simulation and Visualization of Plant Competition
}

\author{
Monssef Alsweis and Oliver Deussen \\ Department of Computer and Information Science \\ University of Konstanz, Germany \\ \{Alsweis, deussen\}@inf.uni-konstanz.de \\ http://graphics.uni-konstanz.de
}

\begin{abstract}
The Wang Tiles method is a successful and effective technique for the representation of $2 \mathrm{D}$-texture or $3 \mathrm{D}$-geometry. In this paper we present a new method to fill Wang tiles with a 2D-FON distribution or a 3D-geometry in order to achieve a more efficient runtime. We extend the Wang Tiles method to include information about their position. We further demonstrate how the individual tiles are filled with different intensities by using the FON distribution. Additionally, we present several new methods to eliminate errors between the tile edges and the different resource areas applying FON and corners relaxation techniques.
\end{abstract}

\section{Introduction}

Modelling and visualization of large complex scenes of plants is a difficult and time consuming task. For example, the storing of plant compositions is extremely difficult because a square kilometer of forest consists of millions of plants, hundred of thousands of small trees, and numerous small scrubs. To model intricate natural scenes, several methods already exist in computer graphics. The primary method was introduced in [1]. A new stochastic algorithm was non-periodically presented to cover the area with a small set of Wang Tiles $[2,3]$. This method provides Wang Tiles with the efficiency of being re-usable tiles, which in turn allows for rendering larger areas of complicated textures, patterns or prelighted geometry at more efficient runtime. Wang Tiles are a set of squares in which each edge of each tile is colored. Matching colored edges are aligned to tile an area. In [1] a set of eight tiles were used to cover an area, and a 2D Poisson Disk distribution was applied to fill the tiles. The aim is to render beautiful and realistic natural scenes. We use four sets or more of tiles instead of one set. Each set of tiles consists of eight tiles. All sets of tiles share the same color coding. However, they differentiate from each other through the intensity of the distributions on the tiles. In the final tiling and scene, rsp., the intensity of the distribution depends on the terrain or on the amount of the resources in which the tiles are positioned. For example, the intensity of the distribution of the plants in an area with poor resources differs from those in the area with rich resources. Applying this method enables to generate large differences in the intensity of the plants in 
two areas. To eliminate this problem, we use several sets and apply some kind of super sampling (antialiasing) method for the selection of the matching sets.

Often plant positions form a 2D Poisson Disc Distribution: each object is presented by a circular area in which are no other objects and in which no other areas overlap. This is done due to what happens in nature when plants prevent other plants to grow in their vicinity. To model this behaviour, we applied the socalled FON (Field-Of-Neighbourhood) distribution model [4]. In the FON model each object is presented in a circular area. The size of this circle depends of the size of the object and of the terrain or the amount of resources.

\section{Previous Works}

There have been different approaches for accelerating of rendering for complex models. Many approaches aimed to avoid visual artifacts which have been repeatedly created using simple methods.

One of these approaches is the rendering of complicated natural scenes through the application of the Wang Tiling Method $[1,2,3]$ in which a limited set of tiles is used to layout a possibly infinite plane. We combined this model with an ecosystem simulation model, the so-called FON (Field Of Neighbourhood) Modell by Berger und Hildenbrandt in [4] and [5]. This model is an individuumbased model and describes a circular zone of the area of influence around the plant. The radius of this area determines the distance in which the neighboring plants influence each other. In [6] the radius of this zone is specified by a nonlinear function which depends from the basal radius of the plant, the size of the plant, the amount of resources needed by the plant, and the area necessary to provide the resources.

Modelling of Complex Ecosystems: In some earlier works [7, 8, 9] nature was rendered using artificial mechanisms. Other scientists $[10,11]$ tried to imitate the actual natural processes. Both approaches are still used and many natural and other complex phenomena were modelled in $[12,13,14]$. In $[15,16,17]$ most important factors that influence the shape, the structure, and the development of a plant were modeled. The first work that simulated the competition and the development of the plants was published by [18]. Lane and Prusinkiewicz extended this work and developed a new method called Multiset L-Systems that was used for the description of the competition between plants. In [19] is described how plants grow in groups, and how the communities compete for resources. In [20], a new method was introduced for the visualization and simulation of the developments of a plant group. In this method, the plant competition for resources was presented as a symmetric and asymmetric competition. Asymmetric competition takes place when plants differ in size or in type.

\section{Our Method}

To tile an area, we apply the Wang Tiling Method. A Wang Tile set consists of square tiles with color-coded edges. The squares cannot be rotated. A valid 


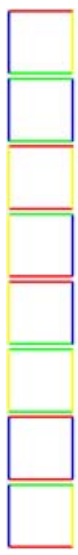

(a)



(b)
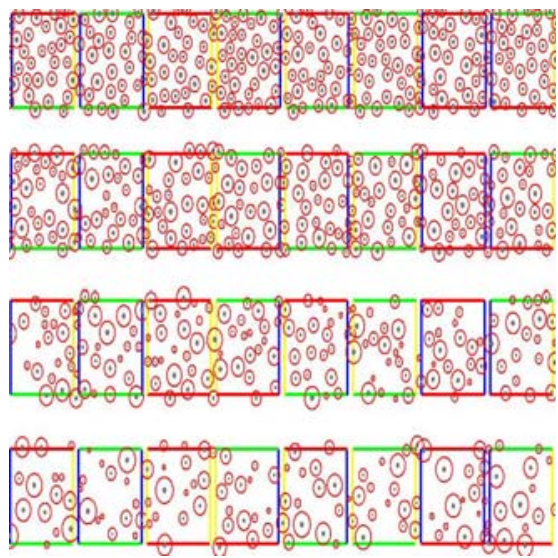

(c)

Fig. 1. a) Tile set; b) Valid tiling; c) four sets of Wang tiles with different distribution intensity

tiling of the infinite plane consists of any number of copies from the set laid down such that all continuous edges have matching colors. (s. Fig. 1).

\subsection{Tiling}

In this work we use four sets or more, each consisting of a set of eight tiles. Each set resembles the other in the color at the edge of the tiles, and differentiates itself from the others through the intensity of the distribution in the tiles. The reason for the use of of four or more sets of tiles with different intensity on the edges is as follows: From Fig. 2(a) it can be seen that the area as well as the different levels of resources differ for different terrains. Consequently, the in the white circles marked tiles from Fig. 2(b) contain different numbers of plants although all marked tiles have the same edge color. In order to receive a soft border between two different resource areas, and at the same time to position

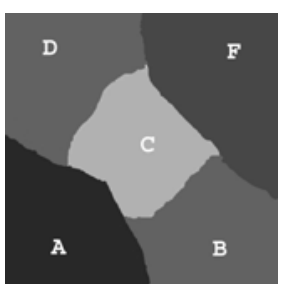

(a)

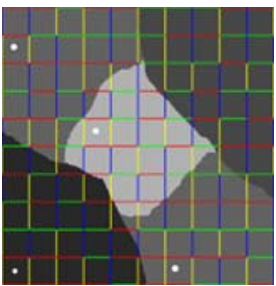

(b)

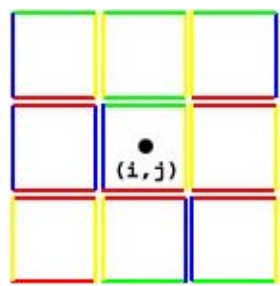

(c)

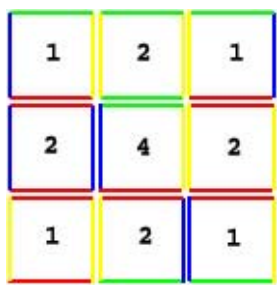

(d)

Fig. 2. (a) area covered with five intensities of the resources; (b) the edges of the resulting tiling match, different intensities are applied; (c) a window is centered around the sampled value $(i, j) ;(\mathrm{d})$ a filter kernel is multiplied with each respective sampled value 
the tiles matching the set of tile $I^{\prime}$ in the area, we apply an image operator for averaging. The intesnsity $I^{\prime}(i, j)$ is computed as:

$$
\frac{1}{M} \sum_{p=S_{i}-k}^{S_{i}+k} \sum_{q=S_{j}-k}^{S_{j}+k} I(p, q) h\left(S_{i}-p, S_{j}-q\right)
$$

Whereby $(i, j)$ indicates the tile in the position $(i, j)$ (see Fig. $2(\mathrm{a})$ ), $S$ is a scaling factor, and $h$ is a filter of the dimension $k$. The selection of the set order is easy to understand and to realize, however, it is computationally expensive. In Fig. 2(a)-(b) a window is centered over an sampled value and a weighted sum of products is achieved by means of multiplication of each sampled value with the respective weight in the filter. The weight can be adjusted in order to implement differing filter kernels. The digital filtering continues in that the window is moved through $n=k * k$ sampling value and the next weighted sum of products is computed.

The use of a $3 \times 3$ window indicates that nine sampled values are part of the final tile computation. On the other hand, the use of a $7 \times 7$ windows includes a computation of 49 integer multiplication. The result of this computational expense is obvious. For a virtual $3 \times 3$ window filter, a filter on tiles in the virtual image can be positioned, in that step-by-step three super-sampling tiles are used. $M$ is computed by the following equation:

$$
M=\sum_{p^{\prime}=0}^{2 k} \sum_{q^{\prime}=0}^{2 k} h\left(p^{\prime}, q^{\prime}\right)
$$

Here, $I$ is the sampling value of the level of the resources in a tile. In order to determine this value in a tile, the resources from different positions in certain tiles are sampled. The average of these sampled resources levels is designated to be the sampled value.

\subsection{FON Distribution}

In order to fill each individual tile with different intensity, we use the already mentioned FON Modell Distribution. In this model each individual plant has a circular zone of influence (ZOI), the radius of the zone determines the distance in which the individual plant influences the neighboring plant.

Each individual plant is identified in the tile through its position, size, and the age. Additionally, each plant has a FON influence zone. To determine the FON influence zone, a non-linear function of the basal radius is applied [4] (s. Fig. 3).

$$
R_{F O N}=a\left(R_{\text {basal }}\right)^{b}
$$

$R_{F O N}$ is the radius of the FON influence zone of the individual plant. $R_{\text {basal }}$ is the radius of the individual plant (Fig. 3). $a$ and $b$ are constants and depend on the intensity of the resources in the ground and of the intensity of the light in above-ground. Typically $a$ has the range of $[12,133]$ and $b \in[1.2,2.3]$. 


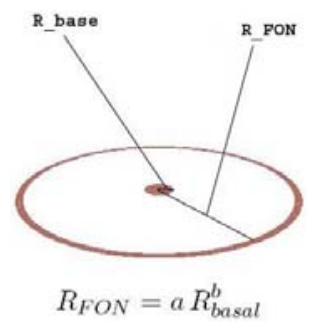

Fig. 3. The FON model. The zone of influence $\left(R_{F O N}\right)$ depends on the diameter of the trunk.

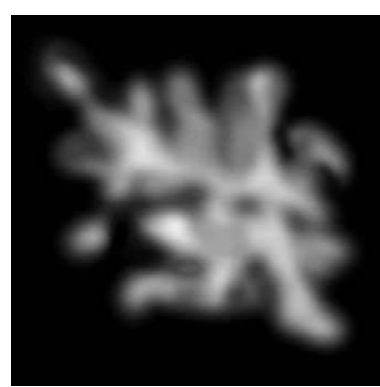

(a)

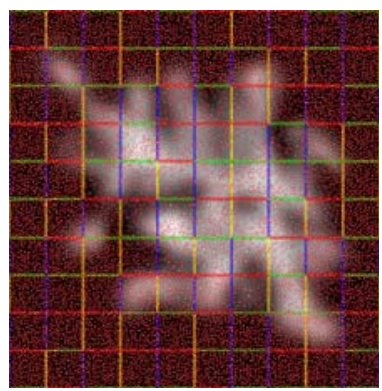

(b)

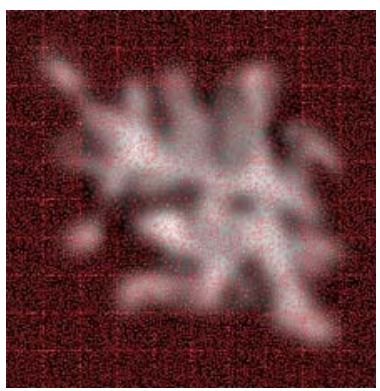

(c)

Fig. 4. In (a) represents the intensity of the resources. In (b) the FON-Tile Method is applied. In $(c)$ shows the noice between the tiles.

Application of the tiles and the FON Distribution in Fig. 4 produces the result in subfigure $(b)$. Subfigure $(a)$ shows an image map of the intensity of the resources in the ground. In $(b)$ is shown that the intensity of the plants in the resource-poor areas is less than in the resource-rich areas. As a result of implementing the averaging, we can derive a gradual change of that the intensity of the plants between two different regions.

\subsection{FON Relaxation}

In Fig. 4(c)we note that an uneven distribution at the tile borders is produced. In order to eliminate this error, we apply the FON relaxation method at the edge of the tiles.

The FON relaxation method is applied to test whether the FON-zone of plants positioned at the edge of one tile overlap with the FON-zone of the plants positioned at the edge of the neighboring tile, and whether the FON-zone of one plant is smaller or larger than that of the neighboring plant at a certain threshold. If the test is positive, the plant must be removed from the respective tile.

In Fig. 5 it is shown that plant number one passed the test and has to be removed from the tile. On the other side the test was not passed for plant number two, and therefore it can remain in the tile. If we compare Fig. 4(c) with 


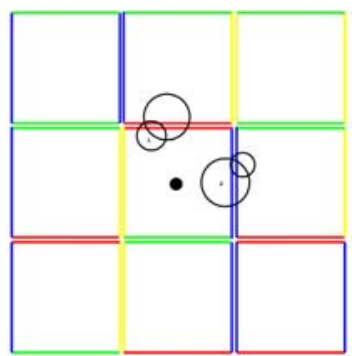

Fig. 5. The circles are presented in two conditions at the edge of the tiles. The first circles is smaller than the other one. The second circle is larger than the other one.

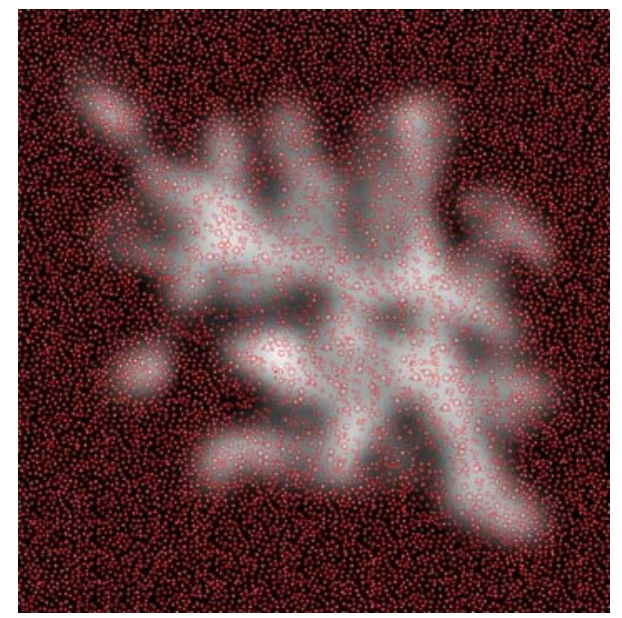

Fig. 6. As a result of implementing the FON relaxation in (c) (Fig. 4), the noise between the tiles was removed.

Fig. 6, we note, that the distribution of the plants is now more even. However, the price is that some small amount of computation has to be done during the tile-based layout.

\subsection{Relaxation of Corners}

From Fig. 7 we note that the difference of the plant intensity between the tile $A$ and the tiles $B, C$ und $D$ creates corners. These corners produces a non-realistic appearance about the appearance of the plants in the scene. To eliminate this unwanted appearance, we apply the relaxation method to tile $A$. The implementation of the this relaxation method is divided into the following steps:

1. It is tested whether tile $A$ produces corners This happens when the intensity in $A$ differs from the intensity in $B, C$ und $D$. 


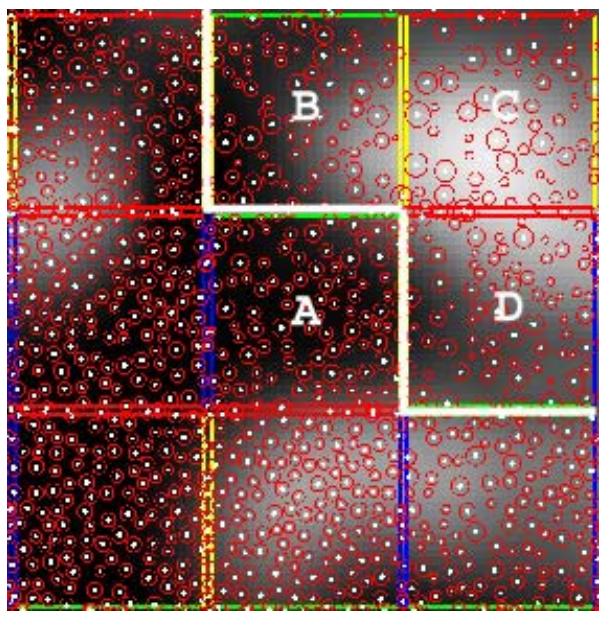

Fig. 7. The intensity corners between the tiles $A$ and tiles $B, C$ and $D$

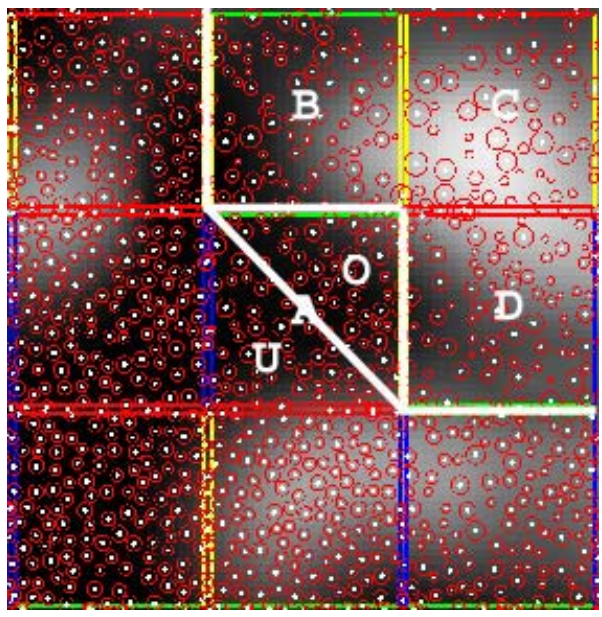

Fig. 8. Distribution of the tiles $A$ into two parts $U$ and $O$

2. The tile $A$ is divided into two diagonal parts (lower part $U$ and upper part $O$ ) in order to be filled with two different intensities. For example as in (Fig. 8).

3. The intensity in part $U$ will not be changed. against it the intensity in part $O$ depends on the intensities in tiles $B, C$ und $D$. The arrangements of $B$, $C$ und $D$ control the definition in the set order in part $U$.

4. Fon-relaxation is applied between diagonal parts.

The application of the last four steps in Fig. 4(c) results in an evenly natural plant distribution without corners in Fig. 6. 


\section{Implementation}

Implementation of this method results in a good quality of a natural scene, so that the noise between the tiles and the noise between the two different resource areas are improved. Additionally, this scene is rendered in real-time. To implement this method, we use a discrete system of several sets. The implementation of these systems can be divided into the following steps:

1. We produce different sets of tiles. Each set differs in plant intensity from the other.

2. each plant is presented in two circles. The first represents the size of the plants, and the second represents the FON-zone of the plant.

3. We apply the Wang-Tiling method for different sets in order to cover the area.

4. In order to eliminate the noise between the tiles, we apply the FON-relaxation method.

5. In order to remove the corners between the tiles, we apply the corner relaxation method.

The plants used in this approach were modelled with the Xfrog Software [21], and were then imported into our systems as POVRAY MESH2 in Fig. 9 and Fig. 10.
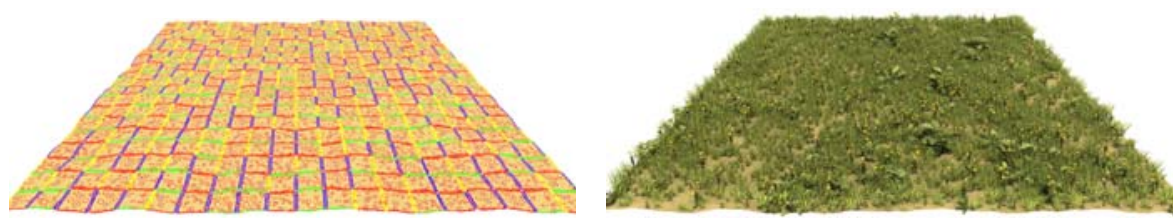

Fig. 9. This image shows the rendering of a scene of a meadow and flowers applying the tiling method with different intensities of resources
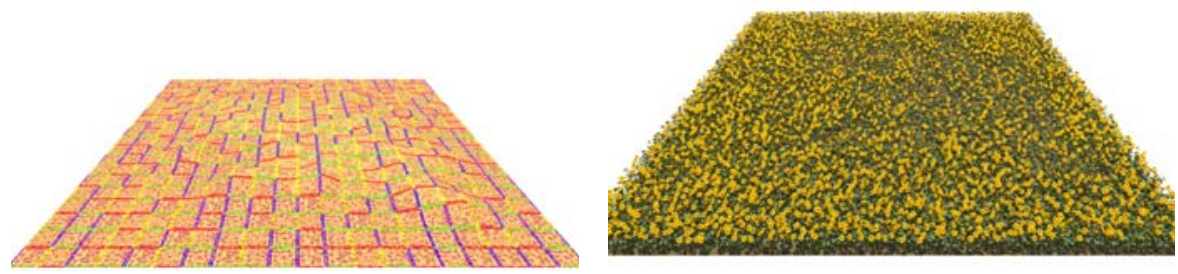

Fig. 10. In this image different resource intensities in a field of sunflowers are shown

\section{Results}

We introduced a fine efficient method to render natural scenes with a more realistic appearance. The use of more sets of tiles with different intensity produces 


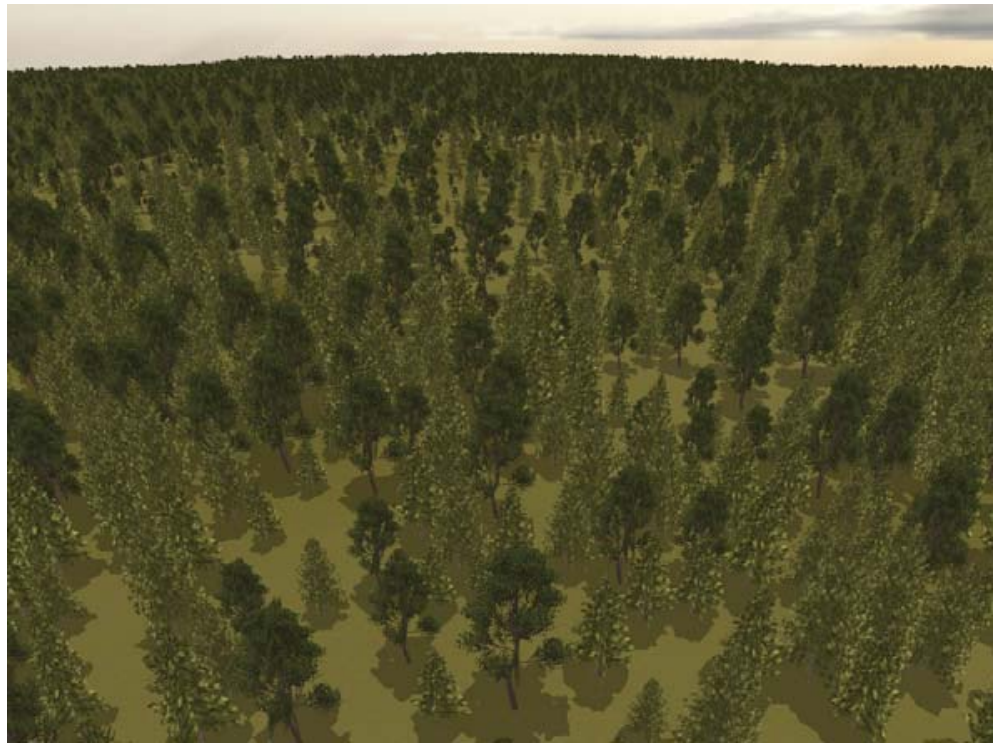

Fig. 11. In this image different resource intensities in African landscape are shown

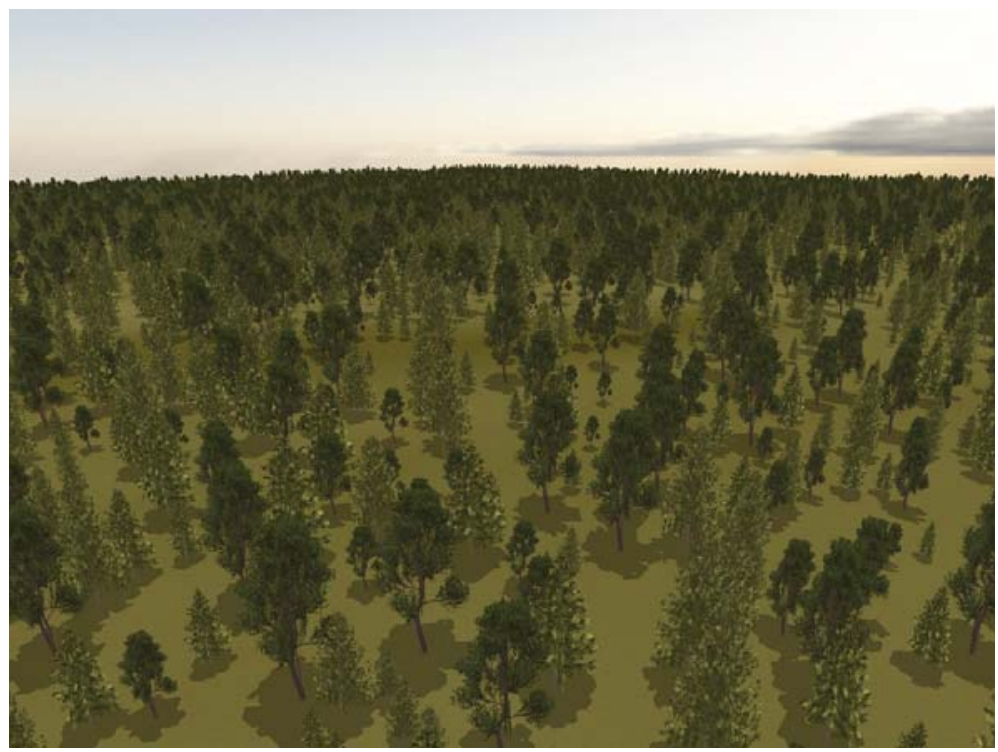

Fig. 12. In this image different resource intensities in African landscape from other camera position are shown 


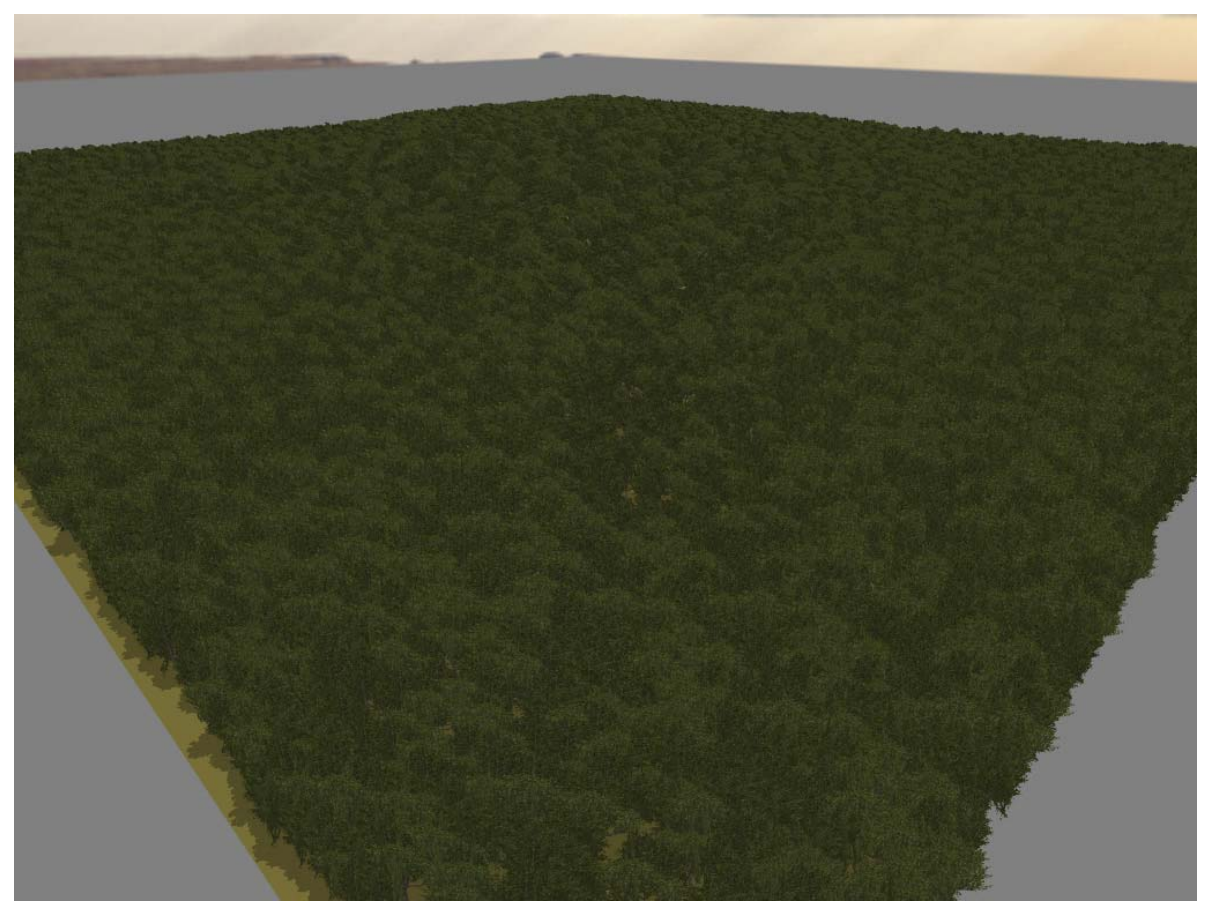

Fig. 13. In this image different resource intensities in Amazon landscape are shown

scenes that have a more natural appearance (see Figs. 9 and 10). The implementation of the FON-relaxation and corner relaxation in one scene produces smooth edges between the tiles and a gradual transformation between two different resource intensities (Figs. 11, 12 and 13).

\section{References}

1. Michael F. Cohen, Jonathan Shade, Stefan Hiller, and Oliver Deussen. Wang tiles for image and texture generation. Proceedings of ACM SIGGRAPH., pages 287294, July 2003.

2. H. WANG. Proving theorems by pattern recognition ii. Bell Systems Technical Journal., 40:142, 1961.

3. H. WANG. Games, logic, and computers. Scientific American (November)., page 98106, 1965.

4. Berger U. and Hildenbrandt H. A new appoarch to spatially explicit modelling of forest dynamics: Spacing, ageing and neighbourhood competition of mangrove trees. Ecological Modelling, 132:287302, 2000.

5. S. Bauer, U. Berger, H. Hildenbrandt, and V. Grimm. Cyclic dynamics in simulated plant populations. Proc R Soc Lond B Biol Sci., 269(1508):2443-2450, 2002.

6. U. Berger, H. Hildenbrandt, and V. Grimm. Towards a standard for the individualbased modeling of plant opulations: self-thinning and the field of neighbourhood approach. Nat. Ressource Model, 15:3954, 2002. 
7. Geoffrey Y.Gardner. Visual simulation of clouds. in proceedings of the 12th annual conference on computer graphics and interactive techniques. ACM Press., page 297304, 1985.

8. Robert Marshall., Rodger Wilson., and Wayne Carlson. Procedure models for generating three-dimensional terrain, in proceedings of the 7th annual conference on computer graphics and interactive techniques. ACM Press., page 154162, 1980.

9. Nelson L. Max. Vectorized procedural models for natural terrain: Waves and islands in the sunset, in proceedings of the 8th annual conference on computer graphics and interactive techniques. ACM Press, page 317324, 1981.

10. Alain Fournier and William T. Reeves. A simple model of ocean waves. in proceedings of the 13th annual conference on computer graphics and interactive techniques. ACM Press, page 7584, 1986.

11. Craig W., Reynolds., Flocks, herds, and schools. A distributed behavioral model, in proceedings of the 14th annual conference on computer graphics and interactive techniques,. ACM Press, page 2534, 1987.

12. Yoav I., H. Parish, and Pascal Muller. Procedural modeling of cities, in proceedings of the 28th annual conference on computer graphics and interactive techniques,. ACM Press, page 301308, 2001.

13. Yoshinori Dobashi., Kazufumi Kaneda., Hideo Yamashita., Tsuyoshi Okita., and Tomoyuki Nishita. A simple, efficient method for realistic animation of clouds,in proceedings of the 27th annual conference on computer graphics and interactive techniques. ACM Press/Addison-Wesley Publishing Co, page 1928, 2000.

14. Paul Fearing. Computer modelling of fallen snow. in proceedings of the 27th annual conference on computer graphics and interactive techniques. ACM Press/Addison-Wesley Publishing Co, page 3746, 2000.

15. J. Arvo and D. Kirk. Modeling plants with environment-sensitive automata. In Proceedings of Ausgraph88, I:2733, 1988.

16. R. Mech and P. Prusinkiewicz. Visual models of plants interacting with their environment. in proceedings of siggraph96. ACM Press, 30(4):397410, 1996.

17. P. Prusinkiewicz, J. Hanan, M. Hammel, , and R. Mech. Lsystems: from the theory to visual models of plants. Machine Graphics and Vision, 2(4)(1508):1222, 1993.

18. O. Deussen, P. Hanrahan, B. Lintermann, R. Mech, M. Pharr, and P. Prusinkiewicz. Realistic modeling and rendering of plant ecosystems. in proceedings of siggraph98, annual conference series 1998. ACM Press, page 275286, 1998.

19. Bedrich Benes. and Juan Miguel Soto Guerrero. Clustering in virtual plant ecosystems. WSCG SHORT Communication papers proceedings., 2004.

20. M. Alsweis and O. Deussen. Modeling and visualisation of symmetric and asymmetric plant competition. Eurographics Workshop on Natural Phenomena., 2005.

21. O. Deussen and B. Lintermann. A modelling method and user interface for creating plants. In Proc. Graphics Interface 97, pages 189-198. Morgan Kaufmann Publishers, May 1997. 